OPEN ACCESS

Edited by:

Xiaobo Mao,

Johns Hopkins University,

United States

Reviewed by:

Xiuli Yang,

The Johns Hopkins Hospital,

United States

Enquan Xu,

Duke University, United States

Longgang Jia

Tianjin University of Science and Technology, China

${ }^{*}$ Correspondence:

Yunhai Tu

23200469@qq.com

Xiaozheng Liu

Ixz_2088@hotmail.com

tThese authors have contributed equally to this work and share first authorship

Specialty section:

This article was submitted to

Neurodegeneration,

a section of the journal

Frontiers in Neuroscience

Received: 16 September 2019

Accepted: 02 December 2019

Published: 14 January 2020

Citation:

Yan J, Wang Y, Miao $\mathrm{H}$ Kwapong WR, Lu Y, Ma Q, Chen W,

Tu $Y$ and Liu $X$ (2020) Alterations in the Brain Structure and Functional

Connectivity in Aquaporin-4 Antibody-Positive Neuromyelitis

Optica Spectrum Disorder.

Front. Neurosci. 13:1362.

doi: 10.3389/fnins.2019.01362

\section{Alterations in the Brain Structure and Functional Connectivity in Aquaporin-4 Antibody-Positive Neuromyelitis Optica Spectrum Disorder}

Jueyue Yan't, Yu Wang ${ }^{2 t}$, Hanpei Miao ${ }^{3}$, William Robert Kwapong³, Yi Lu², Qingkai Ma4, Wei Chen ${ }^{5}$, Yunhai Tu ${ }^{3 *}$ and Xiaozheng Liu ${ }^{2 *}$

' Department of Neurology, The Second Affiliated Hospital \& Yuying Children's Hospital, Wenzhou Medical University, Wenzhou, China, ${ }^{2}$ China-USA Neuroimaging Research Institute, Department of Radiology, The Second Affiliated Hospital \& Yuying Children's Hospital, Wenzhou Medical University, Wenzhou, China, ${ }^{3}$ School of Ophthalmology and Optometry, Wenzhou Medical University, Wenzhou, China, ${ }^{4}$ Department of Opthalmology, The First Affiliated Hospital of Anhui Medical University, Hefei, China, ${ }^{5}$ Department of Psychiatry, Sir Run Run Shaw Hospital, Collaborative Innovation Center for Brain Science, Zhejiang University School of Medicine, Hangzhou, China

Purpose: To investigate the mechanisms underlying the gray matter volume (GMV) and functional connectivity (FC) changes in aquaporin-4 antibody-positive neuromyelitis optica spectrum disorder (NMOSD) patients.

Methods: This cross-sectional study consisted of 21 patients with aquaporin4 antibody-positive NMOSD and 22 age- and sex-matched healthy controls. All participants underwent cerebral magnetic resonance imaging and testing each individual's visual acuity was done.

Results: Neuromyelitis optica spectrum disorder patients showed significantly reduced GMV in the left calcarine, left thalamus and right lingual gyrus of the NMOSD patients when compared to $\mathrm{HC}(P<0.05)$. NMOSD patients showed significantly decreased FC values $(P<0.05)$ in both the left and right calcarine, right lingual gyrus and left thalamus, respectively, when compared to HC. We also observed a positive correlation between the FC values of the left thalamus, bilateral calcarine gyrus and the visual acuity, respectively $(P<0.05)$. Furthermore, a negative association was seen between the duration of the disease, frequency of optic neuritis, and the $\mathrm{FC}$ values in the lingual gyrus, bilateral calcarine gyrus, and right lingual gyrus, respectively $(P<0.05)$.

Conclusion: Reduced visual acuity and frequency of optic neuritis are associated with alterations in the GMV and FC in NMOSD. Our current study, which provides imaging evidence on the impairment involved in NMOSD, sheds light on pathophysiological responses of optic neuritis attack on the brain especially on the visual network.

Keywords: gray matter volume, functional connectivity, neuromyelitis optica spectrum disorder, resting-state functional magnetic resonance imaging, optic neuritis 


\section{INTRODUCTION}

Neuromyelitis Optica spectrum disorders (NMOSD) is a rare autoimmune inflammatory disorder of the central nervous system (CNS) which affects the brain and eye and is characterized by attack(s) of optic neuritis (ON) and myelitis (Jarius et al., 2014). The distinctive clinical features of NMOSD are decreased vision (which may later lead to blindness), pain during eye movement, and visual field defects after attack(s) of optic neuritis (Vabanesi et al., 2019; Wu et al., 2019). Previous reports have stressed on the significantly decreased vision that results from ON in NMOSD; in a study by Wingerchuk et al. (1999), over $50 \%$ of NMOSD patients who were relapsing, were almost blind in at least an eye in the US while a study by Kitley et al. (2012) reported of bilateral permanent visual loss in a portion of NMOSD patients in the United Kingdom and Japan. Patients develop monophasic or recurrent optic neuritis, longitudinally extensive transverse myelitis, and brainstem lesions that can lead to substantial disability and even death (Silbermann and Bourdette, 2019).

Improvement in neuroimaging technology has brought forth both the structural and functional data in brains; currently, NMOSD patients with or without ON can be imaged effortlessly. Voxel-based morphometry (VBM) is a whole-brain morphology analysis that compares the voxel-wise, intra-group differences in local brain morphology (Ashburner and Friston, 2000). A neuroimaging report from Chan et al. (2011) showed significant changes that occurred in the microstructural volume of NMOSD patients when compared to healthy controls (HC). A previous study based on VBM has shown significant loss in gray matter volume (such as the thalamus) in patients with NMOSD after ON when compared to healthy controls (Kim et al., 2015). Furthermore, longitudinal studies using VBM on NMOSD showed significant loss of gray matter especially in patients with recurrent ON attack (Kim et al., 2012; Carnero Contentti et al., 2018). Another study also showed that changes in the microstructural of the gray matter affect the visual network of the brain thus affecting the vision of NMOSD patients (Pittock and Lucchinetti, 2016).

Functional magnetic resonance imaging (fMRI) using a bold oxygenation level-dependent (BOLD) signal is one of the most versatile and vital tools to study non-invasive functional activation of the human brain in vivo. Functional connectivity (FC) in resting-state functional MRI (RS-MRI) reflects the correlation of the blood-oxygen level-dependent signals in different portions of the brain in a time sequence; seed correlation analysis is one of the common FC analysis used. Both VBM and FC methods have been widely used in NMOSD and multiple sclerosis (MS) (Cruz Gomez et al., 2013) studies; however, understanding the structural and functional significance of $\mathrm{ON}$ in the brain of MS disease cascade is limited by the presence of demyelinating lesions in the optic radiation and typically relatively mild visual impairment. In contrast, NMOSD represents a definitive model for the exploration of functional changes in the brain after $\mathrm{ON}$, given the non-appearance of optic radiation injuries in typical cases together with severe damage to the visual system.
Microstructural and functional neuroimaging studies have separately reported the functional and structural alterations associated with NMOSD, yet, the relationship between these microstructural and functional changes remains ambiguous. Benoliel et al. (2017) have shown the association between clinical symptoms and structural and functional cerebral changes in NMOSD but very little is known of the changes that occur in the brain activity and volume after $\mathrm{ON}$ attack(s). We hypothesize that there may be changes in the visual cortex after demyelination of the optic nerve in NMOSD patients after ON attack(s).

Thus our current study combined the VBM method with the seed-based resting-state analysis method to explore the visual cortex and the whole brain FC changes in NMOSD after ON $\operatorname{attack}(\mathrm{s})$ (1) assessing the differences in gray matter volume (GMV) and FC between NMOSD patients and HC, (2) exploring the association between FC measurements and the clinical information, and (3) investigating the mechanisms underlying the FC changes in the frequency of ON attacks.

\section{MATERIALS AND METHODS}

\section{Demographics}

Twenty-five patients diagnosed with NMOSD who met the 2015 international consensus diagnostic criteria of NMOSD (Wingerchuk et al., 1999) were enlisted from the outpatient of the Neurology Department, The Second Affiliated Hospital and Yuying Children's Hospital of Wenzhou Medical University between January 2019 and June 2019. AQP-4 antibody tests were implemented for all patients by the hospital's specialized laboratory. The inclusion criteria for patients were Chinese ethnicity and anti-AQP4 antibody seropositive. Patients who were MOG antibodies positive were excluded. All patients recruited were relapse-free for 6 months or more and without high dose corticosteroid treatment before MRI investigation. Other inclusion criteria included: 1 . Vision loss with or without pain in the eye, 2. Visual field defects associated with damage to the nerve fibers of the eye, 3. Exclusion of other possible diagnoses, such as toxic, genetic, metabolic or invasive optic neuropathy, and 4. Sufficient MR image quality. Four NMOSD patients were excluded after MR imaging due to poor imaging quality. The control group included 22 controls from the hospital workers or interns in the hospital who were age and sex-matched; controls who had no history of neurologic or psychiatric disease and were matched for age and sex. The exclusion criteria were: 1 . No neurological or psychiatric illness, 2. No visual system-related diseases, 3. No abnormality on MR imaging. All participants underwent a baseline clinical MRI; none of the participants were found having brainstem syndromes. The Ethics Committee of The Second Affiliated Hospital and Yuying Children's Hospital of Wenzhou Medical University approved of this study and was in accordance with the Declaration of Helsinki. All participants provided informed consent before enrollment in our study.

\section{Aquaporin-4 Immunofluorescence Assay}

Analysis for serum samples for the presence of AQP-4 antibodies by extracellular live cell-staining immunofluorescence technique 
using transiently transfected AQP-4 expressing cells as previously reported (Mader et al., 2010; Di Pauli et al., 2011). Screening for the detection of AQP-4 was done at dilutions of 1:20 and 1:40 by specialized clinicians.

\section{Measurement of Visual Acuity}

Early Treatment Diabetic Retinopathy Study chart at a 3.2-m distance was used for the measurement of each participant's visual acuity. Each participant underwent both monocular and binocular visual acuity evaluation. For patients with very poor vision, fingers were held before the eye or hand movements were done and later transformed as visual acuity (Schulze-Bonsel et al., 2006).

\section{MRI Data Acquisition}

Siemens 3 Tesla Trio Tim MRI scanner equipped with a 32channel head coil was used to image the brain of each participant. The specifications of the MRI used in our current study have been reported in a previous study (Bai et al., 2019).

\section{MRI Data Preprocessing}

For structural MR images, FSL's standard VBM processing pipeline was implemented. An optimized VBM approach as reported by Good et al. (2001) was implemented with all processing steps carried out by means of open ware FSL version 4.1.5 (Beckmann et al., 2006). The pre-processed structural scans for all scanning sessions for a given subject were first rigidly aligned to the first scan using FSL non-linear registration tool (FLIRT) and a mean image was created. Next, tissue-type segmentation was carried out on the subject mean image using FSL automated segmentation tool (FAST). The resulting graymatter partial volume images were then aligned to MNI152 standard space. The resulting images were averaged to create a study-specific template. The brain-extraction and segmentation steps were then repeated on the rigidly aligned structural scans from each scanning session. The segmented native gray matter images were then non-linearly registered to the template using the transformations calculated from the averaged images. The segmented images were then smoothed with an isotropic Gaussian kernel with a sigma of $3 \mathrm{~mm}$.

All fMRI preprocessing was performed using Statistical Parametric Mapping version 8 (SPM8; Welcome Institute of Cognitive Neurology, London) and Data Processing Assistant for rs-fMRI advanced edition (DPARSFA) ${ }^{2}$ in MATLAB software (R2014a) for the resting-state fMRI (rs-fMRI). The first ten time points of each subject were discarded to allow the signal to reach equilibrium and the participants to adapt to the scanning noise. In sequence, slice timing, head motion correction, and spatial normalization to the standard Montreal Neurological Institute (MNI) template with a resampled voxel size of $3 \mathrm{~mm} \times 3 \mathrm{~mm} \times 3 \mathrm{~mm}$. Subjects with head movement less than $2 \mathrm{~mm}$ translation in any axis or less than two angular rotations in any axis during fMRI scanning were included. After that, the data were detrended to remove the linear trend of time

${ }^{1}$ http://www.fmrib.ox.ac.uk/fsl/

${ }^{2}$ http://www.restfmri.net courses and were band-pass filtered (temporal band-pass filtering is $0.01-0.08 \mathrm{~Hz}$ ). Finally, global signal, white matter signal, and cerebral-spinal fluid signal were regressed out as covariates.

For each seed region, a voxel-wise FC analysis was performed separately for each ROI. The mean time series from all of the voxels within the ROI was used as the seed reference time series, and the Pearson's correlation coefficient (between the average time series for that seed and each voxel in the brain) was computed as the strength of FC. For further statistical analysis, the correlation coefficients were transformed to $z$-values using the Fisher r-to-z transformation to improve the normality of the correlation coefficients (Finke et al., 2018). Thus, a map that represented the FC strength and the seed region (in terms of the $z$-values for each subject) was obtained. Four NMOSD patients were excluded due to poor imaging quality.

\section{FSL Voxel-Based Morphometry (FSL-VBM)}

We performed non-parametric statistical analysis using FSL Randomize with 5,000 permutations and threshold-free cluster enhancement (TFCE) option $(P<0.05)$.

\section{Seed-Based Resting-State Functional Connectivity Analysis}

A two-tailed $t$-test was used for comparisons of the FC between the groups. Correction for multiple comparisons was accomplished with 3dClustSim using analysis of Functional NeuroImages $\left(\mathrm{AFNI}^{3}\right) . P<0.05$ was considered statistically significant. All results were viewed on the MNI T1 template and the p or $T$-value scale is shown on the right of the image.

\section{Statistical Analysis}

Statistical analyses for demographics and clinical measures were performed using SPSS version 21. Multiple linear regression was used to assess the association between FC, gray matter changes, visual acuity, and disease duration, respectively. Independent-sample $t$-tests and the Fisher exact test were used where appropriate.

\section{RESULTS}

\section{Demographics and Clinical Information}

Twenty-one patients with NMOSD (mean age 48.0 years) and 22 healthy controls (mean age 47.78) participated in this study. Significant differences $(P>0.05)$ were not seen in age, BMI, and gender. Significant differences $(P<0.001)$ were seen in the disease duration and best corrected visual acuity (BCVA) between the two groups as shown in Table $\mathbf{1}$.

\section{Comparison of Gray Matter Volume Between Neuromyelitis Optica Spectrum Disorder and Healthy Controls}

Analyses of the GMV showed significantly reduced GMV in the left calcarine ( $P=0.028$; Figure 1A and Table 2), left

${ }^{3}$ http://afni.nimh.nih.gov/afni/ 
TABLE 1 | Demographics and clinical information.

\begin{tabular}{lccc}
\hline & NMOSD & Control group & $\boldsymbol{P}$ \\
\hline Eyes & 21 & 22 & \\
Gender & $21 / 0$ & $22 / 0$ & \\
Age, years & $48.0(12.85)$ & $47.78(11.63)$ & 0.953 \\
BMl & $22.34(2.41)$ & $23.82(2.68)$ & \\
Duration & $5.30(4.50)$ & - & $<0.001$ \\
BCVA & $0.44(0.42)$ & $1.11(0.16)$ & $<0.001$ \\
AL & $23.43(1.14)$ & $23.35(1.13)$ & 0.843 \\
IOP & $13.70(3.51)$ & $12.16(2.70)$ & 0.117 \\
Frequency of ON & & & \\
1 time & $8(28.6 \%)$ & & \\
$\geq 2$ times & $13(57.1 \%)$ & & \\
\end{tabular}

NMOSD, neuromyelitis optica spectrum disorders; BMI, body mass index; $B C V A$, best corrected visual acuity; AL, axial length; IOP, intra-ocular pressure; ON, optic neuritis.

thalamus $(P=0.001$; Figure $\mathbf{1 B}$ and Table 2), and right lingual gyrus $(P=0.005$; Figure $1 \mathrm{C}$ and Table 2$)$ of NMOSD patients when compared to $\mathrm{HC}$. All results of the gray matter volume between NMOSD and HC were shown in the MNI T1 template (Figure 1).

\section{Comparison of Functional Connectivity Between Neuromyelitis Optica Spectrum Disorder and Healthy Controls}

Analyses of the FC showed significantly decreased FC values in the left $[\mathrm{NMOSD}=0.399(0.250): \mathrm{HC}=0.685(0.262) ; P=0.002$; Figure 2A] and right $[\mathrm{NMOSD}=0.552(0.230): \mathrm{HC}=0.824$ (0.240); $P=0.001$; Figure 2A] calcarine gyrus, left thalamus $[$ NMOSD $=0.421$ (0.192): $\mathrm{HC}=0.627(0.140) ; P<0.001$; Figure 2B], and right lingual gyrus [NMOSD $=0.223(0.187)$ : $\mathrm{HC}=0.359$ (0.178); $P=0.019$; Figure 2C] of NMOSD patients when compared to $\mathrm{HC}$.

\section{Association Between Aquaporin-4 Levels and Cerebral Parameters}

GMV change in the left thalamus (Rho $=-0.510, P<0.001$ ) was significantly associated with the AQP-4 levels. FC values in the
TABLE 2 | Group differences between individuals with neuromyelitis optica spectrum disorder (NMOSD) and controls.

\begin{tabular}{lccccc}
\hline Region & Brodmann area & Cluster size, $\mathbf{m m}^{\mathbf{3}}$ & \multicolumn{3}{c}{ MNI coordinates } \\
\cline { 4 - 6 } & & & $\boldsymbol{x}$ & $\boldsymbol{y}$ & $\mathbf{z}$ \\
\hline $\mathrm{R}$ lingual & \multirow{2}{*}{18} & 179 & 14 & -54 & -4 \\
$\mathrm{~L}$ thalamus & & 54 & -2 & -16 & 12 \\
$\mathrm{~L}$ calcarine & 17 & 6 & -6 & -64 & 12 \\
\hline
\end{tabular}

MNI, Montreal Neurological Institute. $R$ lingual, right lingual gyrus. $L$ thalamus, the left thalamus. $L$ calcarine, left calcarine gyrus.

right lingual gyrus ( $\mathrm{Rho}=-0.385, P=0.011)$, left $(\mathrm{Rho}=-0.510$, $P<0.001)$ and right $($ Rho $=-0.482, P=0.001)$ calcarine gyrus, and left thalamus (Rho $=-0.538, P<0.001$ ) were significantly associated with AQP-4 levels.

\section{Association Between Gray Matter Changes and Clinical Information}

Gray matter changes in the left thalamus showed significant correlations with frequency (Rho $=-0.522, P<0.001)$, duration (Rho $=-0.519, P<0.001)$, and visual acuity $($ Rho $=0.444$, $P=0.003)$, respectively.

\section{Association Between Functional Connectivity Changes and Clinical Information}

We observed significant correlations between BCVA and the FC values of the left (Rho $=0.508, P<0.001$; Figure 3A) and right (Rho $=0.472, P=0.001$; Figure $3 \mathrm{~B}$ ) calcarine gyrus and left thalamus (Rho $=0.529, P<0.001$; Figure $3 \mathrm{C}$ ) but not in right lingual gyrus (Rho $=0.259, P=0.094$; Figure 3D), respectively. Furthermore, a negative association was seen between the duration of the disease and the FC values in the right lingual gyrus ( Rho $=-0.356, P=0.019$ ), left $($ Rho $=-0.516, P<0.001)$ and right $($ Rho $=-0.450$, $P=0.002)$ calcarine gyrus, and left thalamus $($ Rho $=-0.505$, $P=0.001)$, respectively. The frequency of optic neuritis was also negatively associated with the FC values in the right lingual
A

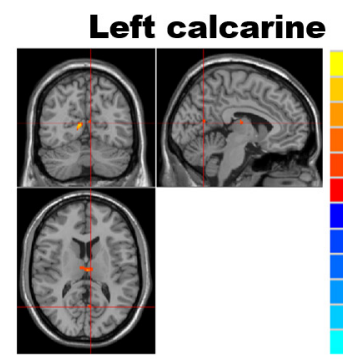

B

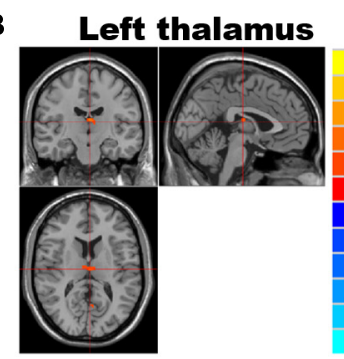

C

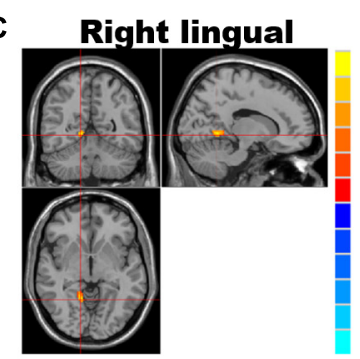

FIGURE 1 | Brain regions showing significantly decreased gray matter volume and group differences (table below) between individuals with neuromyelitis optica spectrum disorder (NMOSD) and controls; from left to right: left calcarine gyrus (A), the left thalamus (B), and right lingual gyrus (C). $(P<0.05$, threshold-free cluster enhancement (TFCE) correction). The gray matter volumes in the left calcarine gyrus (A), left thalamus (B), and right lingual gyrus (C) were significantly reduced $(P<0.05)$ in NMOSD patients when compared to HC. 


\section{A Right lingual}

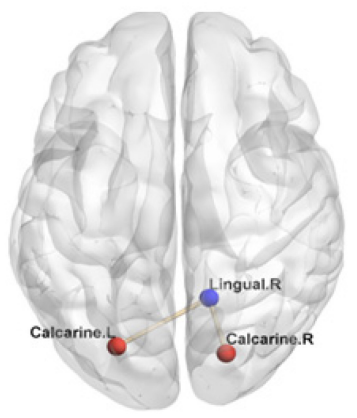

B Left thalamus

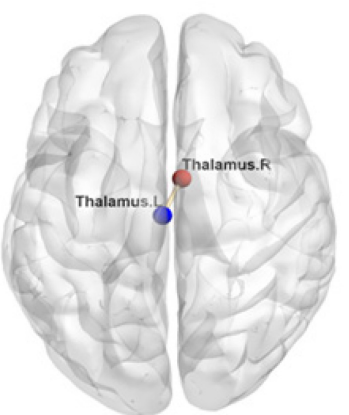

c Left calcarine

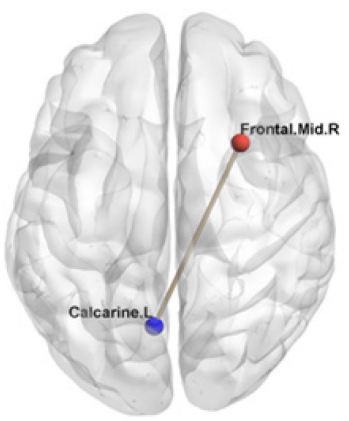

FIGURE 2 | Group differences in functional connectivity (FC) between individuals with neuromyelitis optica spectrum disorder (NMOSD) and controls. The blue dots represent the seed points, and the red dots represent the remaining brain regions displaying differences in FC. The lines represent the functional connections between the seed points and the different brain regions. (A) shows the right lingual gyrus; (B) shows the left thalamus; (C) shows the left calcarine gyrus. The FC values in right lingual gyrus (A), left thalamus (B) and left calcarine gyrus (C) were significantly reduced $(P<0.05)$ in NMOSD patients when compared to HC.

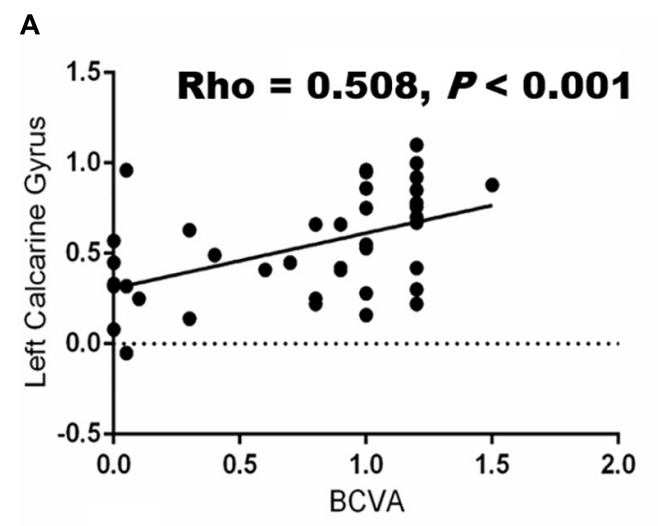

C

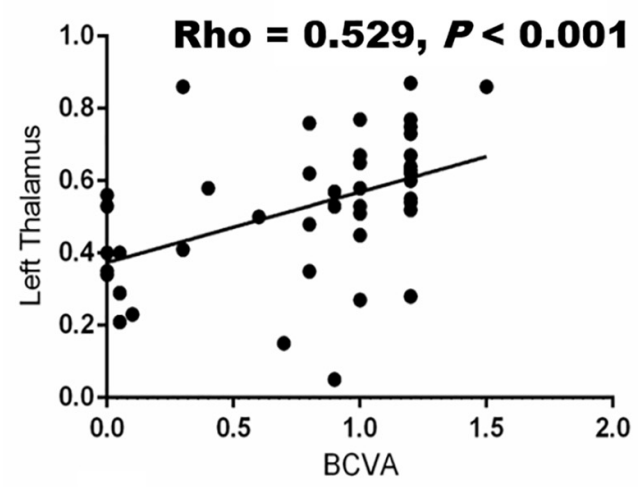

B

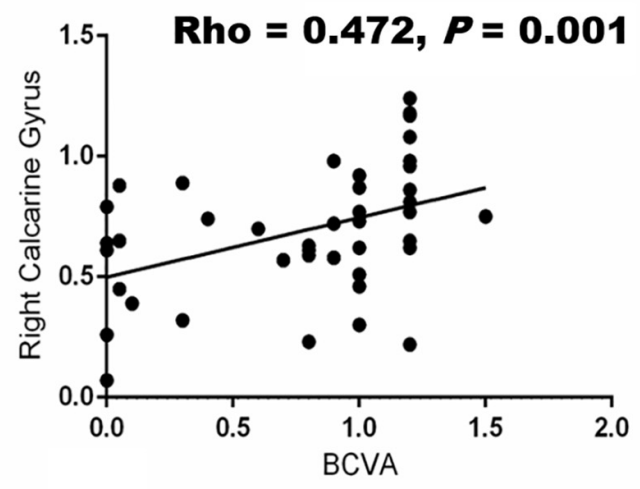

D

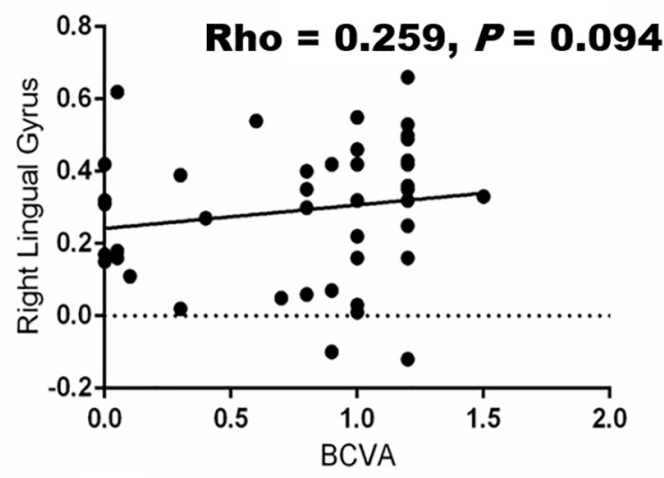

FIGURE 3 | Correlation between BCVA and functional connectivity $(F C)$ values in different brain regions. Significant associations $(P<0.05)$ were shown between BCVA and the changes in FC values of the brain in the left calcarine gyrus (A), right calcarine gyrus (B) left thalamus (C) and right lingual gyrus (D), respectively.

gyrus (Rho $=-0.325, P=0.033$; Table 3$)$, left $($ Rho $=-0.498$, $P=0.001$; Table 3) and right $($ Rho $=-0.565, P<0.001$; Table 3) calcarine gyrus, and left thalamus (Rho $=-0.650, P<0.001$; Table 3), respectively.

\section{DISCUSSION}

Our current study showed that NMOSD patients with AQP-4 antibody-positive are associated with a pronounced decrease in 
TABLE 3 | Correlation between frequency of ON attack and FC values.

\begin{tabular}{lcr}
\hline Regions and BCVA & Rho & $\boldsymbol{P}$ \\
\hline R Lingual & -0.325 & 0.033 \\
R Calcarine & -0.498 & 0.001 \\
L Calcarine & -0.565 & $<0.001$ \\
L Thalamus & -0.650 & $<0.001$ \\
ON & -0.751 & $<0.001$ \\
\hline
\end{tabular}

gray matter and FC in the left thalamus, left calcarine, and right lingual gyrus, respectively, which are constituents of the visual cortex. Furthermore, we showed that changes in the FC were associated with disease duration, visual acuity, and frequency of ON attack, respectively.

A previous report on brain MRI suggested unremarkable changes or non-specific injury in most patients with NMOSD (Kim et al., 2015). On the other hand, the occurrence, clinical manifestations, and changes in cerebral imaging in NMOSD have been reported to be associated with the ethnicity of the patient and the presence of AQP-4 antibody (CabreraGomez et al., 2007, 2008; Pandit et al., 2015). Liu et al. (2015) showed that gray matter damage is a common phenomenon in Chinese patients with NMOSD; however, very little is known of gray matter changes in NMOSD after ON attack(s). Our current study explored a consistent study sample that included AQP-4 antibody-positive NMOSD after ON attack(s) who are Chinese.

AQP-4 antibody has been reported to be expressed in the brain (Badaut et al., 2007); it is the most abundant water channel in the brain (Rash et al., 1998) and controls brain water homeostasis (Nielsen et al., 1997). Amassing reports (Misu et al., 2013; Nishiyama et al., 2016; Yick et al., 2018) have shown that AQP-4 can induce complement independent pathologies. Experimental reports have shown that inflammatory sequela follows the binding of NMO-IgG to AQP-4 (Hinson et al., 2012; Pittock et al., 2013). Kim et al. (2012) suggested that brain MRI abnormalities in AQP-4 autoimmunity are typically localized in the periependymal portions where AQP4 is highly expressed. Furthermore, reports (Viegas et al., 2009; Samart and Phanthumchinda, 2010) have shown that hypothalamic lesions tend to be more extensive in seropositive AQP-4 NMO patients. Our current report showed that gray matter damage was more severe in the hypothalamus, and a significant association was seen between GMV and AQP4 levels; thus, we suggest that AQP-4 may contribute to gray matter damage in the brain, which is congruent with aforementioned reports.

Functional MRI is a neuroimaging modality that processes neural activity changes in deoxyhemoglobin levels (blood oxygen level-dependent [BOLD] signal). Thus far, two common methodologies have been suggested: (1) activation fMRI, which evaluates deoxyhemoglobin signal modification during specified tasks, and (2) resting-state fMRI, which associates the synchrony of low-frequency fluctuations of the BOLD signal in various regions while the brain is at rest; accordingly, this technique has been used to determine the FC of neural networks in the brain (Schmidt et al., 2017). Using fMRI, Rocca et al. (2004) showed an irregular form of movement associated with cortical activation in patients with NMOSD that extended beyond the sensorimotor network and involved the visual network of the brain. Previous studies (Lopes et al., 2015; Cai et al., 2017) have also explored visual cortex FC in NMOSD but did not give an account on its association with the clinical data such as their disease duration or frequency of optic neuritis attack; two previous reports (Lopes et al., 2015; Finke et al., 2018) used the activation fMRI approach and showed increased connectivity in NMOSD patients when compared to healthy controls. Cai et al. (2017) used the seed-based approach, similar to our current approach, and showed decreased connectivity in the higher visual cortex. Our current study showed reduced FC values in both the left and right calcarine, right lingual gyrus, and left thalamus; these regions are vital constituents of the visual cortex and visual pathways; thus, we suggest that there is functional damage in the visual network in NMOSD patients.

Our current study showed significantly reduced gray matter volume in the left thalamus, left calcarine, and right lingual gyrus (which are constituents of the primary visual network). Visual impairment, a key clinical manifestation in NMOSD after ON, has been reported to affect the quality of life (Schmidt et al., 2017) since it may lead to blindness (Collongues et al., 2010; Kitley et al., 2012). Thinning of the visual cortex and impaired microstructural integrity restricted to the optic radiation are consistent neuroimaging reports shown in patients with NMOSD (Matthews et al., 2015; Pache et al., 2016). Significant changes in the GMV and FC of the left thalamus suggest that the thalamus, which has been reported to be the central hub in the brain (Usrey and Alitto, 2015), plays a vital role in the frequency of ON attack in NMOSD. The thalamus is an important relay of motor and sensory information to and from the cerebral cortex; additionally, projections to the primary visual cortex are via the thalamus, emphasizing its importance in the visual network (Saalmann and Kastner, 2011). A previous report (Tona et al., 2014) on the structural and functional thalamic changes with MS using RS-MRI suggested that increased connectivity in the thalamus could reflect the same disease-associated functional change with demyelination disease. The decreased connectivity in the constituents of the primary visual cortex and the thalamus suggests that the observed changes lead to impoverished visual input due to a large portion (thalamus and other constituents of the visual cortex) of the primary visual cortex being affected. A large portion of the primary visual network with decreased FC suggests that functional reorganization may not be able to compensate the functional damage in the intermittent $\mathrm{ON}$ attack in NMOSD patients.

Our current study also showed a high correlation between BCVA and the FC values of the left thalamus and bilateral calcarine gyrus, depicting that changes in the visual network connectivity could be driven by reduced visual input. As such, decreased FC could reflect an attempted compensatory or noncompensatory mechanism of network reorganization in response to the impaired vision in NMOSD. A previous report (Filippi et al., 2013) on MS suggested that increased FC can compensate 
for structural damage in the brain. On the other hand, the relationship between the changes in the constituents of visual cortex FC and decreased visual acuity could be symptomatic of maladaptive process (Schoonheim et al., 2015). In our current study, we suggest that poor visual input might cause a comparable loss of diversity in visual network connectivity patterns in NMOSD, resulting in an unreliable FC change. Longitudinal studies are needed to validate our hypothesis.

A significant correlation was seen between the $\mathrm{FC}$ values and the frequency of ON attack. A previous report (Huang et al., 2018) has shown that recurrent attacks of ON lead to both structural changes and functional alterations of connectivity in the visual network. Our current report echoes what has been previously reported by suggesting that the higher the frequency of ON, the more decreased the FC values will be.

There are some limitations in our current study. First, this study is a cross-sectional study which, although it showed a strong association between FC and impaired visual acuity and disease duration, does not allow the delineation of the underlying causal association. Secondly, the study had a relatively small sample size with limited functional visual measures such as the Snellen chart for visual acuity measurement. The incidence of NMOSD is relatively low (0.5/100000-10/100000) (CabreraGomez et al., 2009; Asgari et al., 2011; Mealy et al., 2012; Flanagan et al., 2016) in China and Asia as a whole when compared to the western world. Wenzhou is a third-class town in China, and most patients decide to visit big cities for treatment which is one of the reasons why we presented with small sample size. Furthermore, our inclusion criteria were quite strict which prohibited the inclusion of four NMOSD patients because they could not meet our inclusion criteria. Our study enrolled 25 NMOSD patients, and 21 were used in our data analyses. Although our sample size is relatively small, our results showed some significant statistical differences when both groups were compared (NMOSD patients and healthy controls). We are currently doing a follow-up on our current study and cooperating with bigger hospitals in larger cities. Further studies with a larger sample size and comprehensive clinical information will be needed to elucidate the associations between the FC measurement and clinical parameters in NMOSD.

\section{REFERENCES}

Asgari, N., Lillevang, S. T., Skejoe, H. P., Falah, M., Stenager, E., and Kyvik, K. O. (2011). A population-based study of neuromyelitis optica in Caucasians. Neurology 76, 1589-1595. doi: 10.1212/WNL.0b013e318219 0f74

Ashburner, J., and Friston, K. J. (2000). Voxel-based morphometry-the methods. Neuroimage 11, 805-821.

Badaut, J., Brunet, J. F., and Regli, L. (2007). Aquaporins in the brain: from aqueduct to "multi-duct". Metab. Brain Dis. 22, 251-263.

Bai, G., Bai, L., Cao, J., Sun, C., Wang, S., Yin, B., et al. (2019). Sex differences in cerebral perfusion changes after mild traumatic brain injury: longitudinal investigation and correlation with outcome. Brain Res. 1708, 93-99. doi: 10. 1016/j.brainres.2018.12.018

Beckmann, C. F., Jenkinson, M., Woolrich, M. W., Behrens, T. E., Flitney, D. E., Devlin, J. T., et al. (2006). Applying FSL to the FIAC data: model-based and

\section{CONCLUSION}

In conclusion, our current study found that NMOSD patients with a history of ON attacks have reduced FC and GMV when compared to healthy controls, and these changes in the brain are associated with their disease duration and frequency of $\mathrm{ON}$ attack. Our current study sheds light on the neural mechanism of ON in NMOSD and longitudinal studies with larger sample sizes are needed to validate our hypotheses.

\section{DATA AVAILABILITY STATEMENT}

All datasets generated for this study are included in the article/supplementary material.

\section{ETHICS STATEMENT}

The studies involving human participants were reviewed and approved by the Ethics Committee of The Second Affiliated Hospital and Yuying Children's Hospital of Wenzhou Medical University. The patients/participants provided their written informed consent to participate in this study.

\section{AUTHOR CONTRIBUTIONS}

All authors listed have made a substantial, direct and intellectual contribution to the work, and approved it for publication.

\section{FUNDING}

This research was supported by the Doctoral Scientific Research Foundation of The Second Affiliated Hospital, Wenzhou Medical University and the General Project of the Department of Science and Technology of Zhejiang Province (2020358406) (XL); the National Key Research \& Development Program of China (2017YFC1310502); and the NSFC Projects of International Cooperation and Exchanges (8161101010) (WC).

model-free analysis of voice and sentence repetition priming. Hum. Brain Mapp. 27, 380-391.

Benoliel, T., Raz, N., Ben-Hur, T., and Levin, N. (2017). Cortical functional modifications following optic neuritis. Mult. Scler. 23, 220-227. doi: 10.1177/ 1352458516649677

Cabrera-Gomez, J., Saiz-Hinarejos, A., Graus, F., Gonzalez-Quevedo, A., Rodriguez-Rojas, R., Quevedo-Sotolongo, L., et al. (2008). Brain magnetic resonance imaging findings in acute relapses of neuromyelitis optica spectrum disorders. Mult. Scler. 14, 248-251. doi: 10.1177/1352458507082617

Cabrera-Gomez, J. A., Kurtzke, J. F., Gonzalez-Quevedo, A., and Lara-Rodriguez, R. (2009). An epidemiological study of neuromyelitis optica in Cuba. J. Neurol. 256, 35-44. doi: 10.1007/s00415-009-0009-0

Cabrera-Gomez, J. A., Quevedo-Sotolongo, L., Gonzalez-Quevedo, A., Lima, S., Real-Gonzalez, Y., Cristofol-Corominas, M., et al. (2007). Brain magnetic resonance imaging findings in relapsing neuromyelitis optica. Mult. Scler. 13, $186-192$. 
Cai, H., Zhu, J., Zhang, N., Wang, Q., Zhang, C., Yang, C., et al. (2017). Subregional structural and connectivity damage in the visual cortex in neuromyelitis optica. Sci. Rep. 7:41914. doi: 10.1038/srep41914

Carnero Contentti, E., Daccach Marques, V., de Castillo, I. Soto, Tkachuk, V., Barreira, A. Antunes, Armas, E., et al. (2018). Frequency of brain MRI abnormalities in neuromyelitis optica spectrum disorder at presentation: a cohort of Latin American patients. Mult. Scler. Relat. Disord. 19, 73-78. doi: 10.1016/j.msard.2017.11.004

Chan, K. H., Tse, C. T., Chung, C. P., Lee, R. L., Kwan, J. S., Ho, P. W., et al. (2011). Brain involvement in neuromyelitis optica spectrum disorders. Arch. Neurol. 68, 1432-1439. doi: 10.1001/archneurol.2011.249

Collongues, N., Marignier, R., Zephir, H., Papeix, C., Blanc, F., Ritleng, C., et al. (2010). Neuromyelitis optica in France: a multicenter study of 125 patients. Neurology 74, 736-742. doi: 10.1212/WNL.0b013e3181d31e35

Cruz Gomez, A. J., Ventura Campos, N., Belenguer, A., Avila, C., and Forn, C. (2013). Regional brain atrophy and functional connectivity changes related to fatigue in multiple sclerosis. PLoS One 8:e77914. doi: 10.1371/journal.pone. 0077914

Di Pauli, F., Mader, S., Rostasy, K., Schanda, K., Bajer-Kornek, B., Ehling, R., et al. (2011). Temporal dynamics of anti-MOG antibodies in CNS demyelinating diseases. Clin. Immunol. 138, 247-254. doi: 10.1016/j.clim.2010.11.013

Filippi, M., Agosta, F., Spinelli, E. G., and Rocca, M. A. (2013). Imaging resting state brain function in multiple sclerosis. J. Neurol. 260, 1709-1713. doi: 10. 1007/s00415-012-6695-z

Finke, C., Zimmermann, H., Pache, F., Oertel, F. C., Chavarro, V. S., Kramarenko, Y., et al. (2018). Association of visual impairment in neuromyelitis optica spectrum disorder with visual network reorganization. JAMA Neurol. 75, 296303. doi: 10.1001/jamaneurol.2017.3890

Flanagan, E. P., Cabre, P., Weinshenker, B. G., Sauver, J. S., Jacobson, D. J., Majed, M., et al. (2016). Epidemiology of aquaporin-4 autoimmunity and neuromyelitis optica spectrum. Ann. Neurol. 79, 775-783. doi: 10.1002/ana. 24617

Good, C. D., Johnsrude, I. S., Ashburner, J., Henson, R. N., Friston, K. J., and Frackowiak, R. S. (2001). A voxel-based morphometric study of ageing in 465 normal adult human brains. Neuroimage 14, 21-36.

Hinson, S. R., Romero, M. F., Popescu, B. F., Lucchinetti, C. F., Fryer, J. P., Wolburg, H., et al. (2012). Molecular outcomes of neuromyelitis optica (NMO)IgG binding to aquaporin-4 in astrocytes. Proc. Natl. Acad. Sci. U.S.A. 109, 1245-1250. doi: 10.1073/pnas.1109980108

Huang, J., Duan, Y., Liu, S., Liang, P., Ren, Z., Gao, Y., et al. (2018). Altered brain structure and functional connectivity of primary visual cortex in optic neuritis. Front. Hum. Neurosci. 12:473. doi: 10.3389/fnhum.2018.00473

Jarius, S., Wildemann, B., and Paul, F. (2014). Neuromyelitis optica: clinical features, immunopathogenesis and treatment. Clin. Exp. Immunol. 176, 149164. doi: $10.1111 /$ cei.12271

Kim, H. J., Paul, F., Lana-Peixoto, M. A., Tenembaum, S., Asgari, N., Palace, J., et al. (2015). Foundation, and Biorepository, MRI characteristics of neuromyelitis optica spectrum disorder: an international update. Neurology 84, 1165-1173. doi: 10.1212/WNL.0000000000001367

Kim, W., Kim, S. H., Huh, S. Y., and Kim, H. J. (2012). Brain abnormalities in neuromyelitis optica spectrum disorder. Mult. Scler. Int. 2012:735486. doi: $10.1155 / 2012 / 735486$

Kitley, J., Leite, M. I., Nakashima, I., Waters, P., McNeillis, B., Brown, R., et al. (2012). Prognostic factors and disease course in aquaporin-4 antibody-positive patients with neuromyelitis optica spectrum disorder from the United Kingdom and Japan. Brain 135, 1834-1849. doi: 10.1093/brain/aws109

Liu, Y., Fu, Y., Schoonheim, M. M., Zhang, N., Fan, M., Su, L., et al. (2015). Structural MRI substrates of cognitive impairment in neuromyelitis optica. Neurology 85, 1491-1499. doi: 10.1212/WNL.0000000000002067

Lopes, F. C., Alves-Leon, S. V., Godoy, J. M., de Souza Batista, S., Scherpenhuijzen, S., Fezer, L., et al. (2015). Optic neuritis and the visual pathway: evaluation of neuromyelitis optica spectrum by resting-state fMRI and diffusion tensor MRI. J. Neuroimaging 25, 807-812. doi: 10.1111/jon.12191

Mader, S., Lutterotti, A., Di Pauli, F., Kuenz, B., Schanda, K., Aboul-Enein, F., et al. (2010). Patterns of antibody binding to aquaporin-4 isoforms in neuromyelitis optica. PLoS One 5:e10455. doi: 10.1371/journal.pone.0010455

Matthews, L., Kolind, S., Brazier, A., Leite, M. I., Brooks, J., Traboulsee, A., et al. (2015). Imaging surrogates of disease activity in neuromyelitis optica allow distinction from multiple sclerosis. PLoS One 10:e0137715. doi: 10.1371/ journal.pone.0137715

Mealy, M. A., Wingerchuk, D. M., Greenberg, B. M., and Levy, M. (2012). Epidemiology of neuromyelitis optica in the United States: a multicenter analysis. Arch. Neurol. 69, 1176-1180. doi: 10.1001/archneurol.2012.314

Misu, T., Hoftberger, R., Fujihara, K., Wimmer, I., Takai, Y., Nishiyama, S., et al. (2013). Presence of six different lesion types suggests diverse mechanisms of tissue injury in neuromyelitis optica. Acta Neuropathol. 125, 815-827. doi: 10.1007/s00401-013-1116-7

Nielsen, S., Nagelhus, E. A., Amiry-Moghaddam, M., Bourque, C., Agre, P., and Ottersen, O. P. (1997). Specialized membrane domains for water transport in glial cells: high-resolution immunogold cytochemistry of aquaporin- 4 in rat brain. J. Neurosci. 17, 171-180.

Nishiyama, S., Misu, T., Nuriya, M., Takano, R., Takahashi, T., Nakashima, I., et al. (2016). Complement-dependent and -independent aquaporin 4antibody-mediated cytotoxicity in human astrocytes: pathogenetic implications in neuromyelitis optica. Biochem. Biophys. Rep. 7, 45-51. doi: 10.1016/j.bbrep. 2016.05.012

Pache, F., Zimmermann, H., Finke, C., Lacheta, A., Papazoglou, S., Kuchling, J., et al. (2016). Brain parenchymal damage in neuromyelitis optica spectrum disorder - A multimodal MRI study. Eur. Radiol. 26, 4413-4422.

Pandit, L., Asgari, N., Apiwattanakul, M., Palace, J., Paul, F., and Leite, M. I. (2015). G.I.C. Consortium, and O. Biorepository for Neuromyelitis, Demographic and clinical features of neuromyelitis optica: a review. Mult. Scler. 21, 845-853.

Pittock, S. J., Lennon, V. A., McKeon, A., Mandrekar, J., Weinshenker, B. G., Lucchinetti, C. F., et al. (2013). Eculizumab in AQP4-IgG-positive relapsing neuromyelitis optica spectrum disorders: an open-label pilot study. Lancet Neurol. 12, 554-562. doi: 10.1016/S1474-4422(13)70076-0

Pittock, S. J., and Lucchinetti, C. F. (2016). Neuromyelitis optica and the evolving spectrum of autoimmune aquaporin-4 channelopathies: a decade later. Ann. N. Y. Acad. Sci. 1366, 20-39. doi: 10.1111/nyas.12794

Rash, J. E., Yasumura, T., Hudson, C. S., Agre, P., and Nielsen, S. (1998). Direct immunogold labeling of aquaporin-4 in square arrays of astrocyte and ependymocyte plasma membranes in rat brain and spinal cord. Proc. Natl. Acad. Sci. U.S.A 95, 11981-11986.

Rocca, M. A., Agosta, F., Mezzapesa, D. M., Falini, A., Martinelli, V., Salvi, F., et al. (2004). A functional MRI study of movement-associated cortical changes in patients with Devic's neuromyelitis optica. Neuroimage 21, 1061-1068.

Saalmann, Y. B., and Kastner, S. (2011). Cognitive and perceptual functions of the visual thalamus. Neuron 71, 209-223. doi: 10.1016/j.neuron.2011. 06.027

Samart, K., and Phanthumchinda, K. (2010). Neuromyelitis optica with hypothalamic involvement: a case report. J. Med. Assoc. Thai 93, 505-509.

Schmidt, F., Zimmermann, H., Mikolajczak, J., Oertel, F. C., Pache, F., Weinhold, M., et al. (2017). Severe structural and functional visual system damage leads to profound loss of vision-related quality of life in patients with neuromyelitis optica spectrum disorders. Mult. Scler. Relat. Disord. 11, 45-50. doi: 10.1016/j. msard.2016.11.008

Schoonheim, M. M., Meijer, K. A., and Geurts, J. J. (2015). Network collapse and cognitive impairment in multiple sclerosis. Front. Neurol. 6:82.

Schulze-Bonsel, K., Feltgen, N., Burau, H., Hansen, L., and Bach, M. (2006). Visual Acuities "Hand Motion" and "Counting Fingers" Can Be Quantified with the Freiburg Visual Acuity Test. Invest. Ophth. Vis. Sci. 47, 1236-1240.

Silbermann, E., and Bourdette, D. (2019). A new era for neuromyelitis optica spectrum disorder. Lancet 394, 1304-1305.

Tona, F., Petsas, N., Sbardella, E., Prosperini, L., Carmellini, M., Pozzilli, C., et al. (2014). Multiple sclerosis: altered thalamic resting-state functional connectivity and its effect on cognitive function. Radiology 271, 814-821. doi: 10.1148/radiol. 14131688

Usrey, W. M., and Alitto, H. J. (2015). Visual Functions of the Thalamus. Annu. Rev. Vis. Sci. 1, 351-371. doi: 10.1146/annurev-vision-082114-035920

Vabanesi, M., Pisa, M., Guerrieri, S., Moiola, L., Radaelli, M., Medaglini, S., et al. (2019). In vivo structural and functional assessment of optic nerve damage in neuromyelitis optica spectrum disorders and multiple sclerosis. Sci. Rep. 9:10371. doi: 10.1038/s41598-019-46251-3

Viegas, S., Weir, A., Esiri, M., Kuker, W., Waters, P., Leite, M. I., et al. (2009). Symptomatic, radiological and pathological involvement of the hypothalamus 
in neuromyelitis optica. J. Neurol. Neurosurg. Psychiatry 80, 679-682. doi: 10. 1136/jnnp.2008.157693

Wingerchuk, D. M., Hogancamp, W. F., O’Brien, P. C., and Weinshenker, B. G. (1999). The clinical course of neuromyelitis optica (Devic's syndrome). Neurology 53, 1107-1114.

Wu, Y., Zhong, L., and Geng, J. (2019). Visual impairment in neuromyelitis optica spectrum disorders (NMOSD). J. Chem. Neuroanat. 97, 66-70.

Yick, L. W., Ma, O. K., Ng, R. C., Kwan, J. S., and Chan, K. H. (2018). Aquaporin-4 autoantibodies from neuromyelitis optica spectrum disorder patients induce complement-independent immunopathologies in mice. Front. Immunol. 9:1438. doi: 10.3389/fimmu.2018.01438
Conflict of Interest: The authors declare that the research was conducted in the absence of any commercial or financial relationships that could be construed as a potential conflict of interest.

Copyright (c) 2020 Yan, Wang, Miao, Kwapong, Lu, Ma, Chen, Tu and Liu. This is an open-access article distributed under the terms of the Creative Commons Attribution License (CC BY). The use, distribution or reproduction in other forums is permitted, provided the original author(s) and the copyright owner(s) are credited and that the original publication in this journal is cited, in accordance with accepted academic practice. No use, distribution or reproduction is permitted which does not comply with these terms. 